\title{
Corona: Kritik in der Krise
}

\begin{abstract}
Zusammenfassung
Dieser Beitrag setzt sich kritisch mit der Kritik linker Theoretiker (v. a. Giorgio Agamben, Byung-Chul Han) an den politischen Maßnahmen zur Eindämmung der Pandemie auseinander, die in der Pandemie nur noch das Wirken einer dystopischen Biopolitik erkennen können. Ich zeige auf, wie in diesen Formen der Kritik ein Jargon der Eigentlichkeit (,Ausnahmezustand") zum Tragen kommt, der die eigene Theoriesprache bestätigt, ohne sich von empirischen Eigenheiten der Pandemie erschüttern zu lassen. Gegen diese „unkritische“ Kritik plädiere ich für mehr Nachdenklichkeit.
\end{abstract}

Schlüsselworte: Kritik, Krise, Theorie, Ausnahmezustand, Pandemie, Nachdenklichkeit

\begin{abstract}
In this paper, I formulate a critique of the critical interventions of left theorists (esp. Giorgio Agamben, Byung-Chul Han), who claimed that the political measure to confine the pandemic were simply the work of dystopian global biopolitics. I show how these forms of critique are mobilizing a jargon of authenticity ("state of exception"), which confirms the propositional language of Theory, but is unable to absorb the unsettling empirical specifics of the current pandemic. Against this "uncritical" critique, I propose a disposition of thoughtfulness.
\end{abstract}

Keywords: critique, crisis, theory, state of exception, pandemic, thoughtfulness

\section{I.}

Verschwörungstheorien zur globalen Pandemie sind populär. Bill Gates soll es gewesen sein. Oder ein deep state, der die Pandemie nutzt, uns alle zwangsimpfen zu lassen. Oder oder oder: „Unglaublich, wie der Blitz des Unsinns in den naiven Volksboden einschlagen und zur politischen Macht werden kann", schreibt dazu Konrad Liessmann (Liessmann, 2020, S. 9). Verschwörungstheorien verbreiten sich rasend schnell, nicht nur in den social media; auch auf Demonstrationen gegen die Pandemiemaßnahmen zeigen Plakate ihre Parolen. Ähnlich schnell, wie sich die Verschwörungstheorien verbreiten, stehen allerdings auch schon die aufgeklärten Gegner/-innen parat, um aus deren Absurdität kulturelles Kapital zu schlagen: Die Argumente der Verschwörungstheoretiker/-innen lassen sich leicht entkräften. An die Stelle des inhaltlichen tritt das emanzipatorische Anliegen, die Verschwörungstheoretiker/-innen von ihrer Dummheit zu befreien: „Argumentative Auseinandersetzungen erübrigen sich, an ihre Stelle tritt die pädagogische Besorgnis" (Liessmann, ebd.).

Schwieriger wird die Situation, wenn die Aussagen aufgeklärter Gegner/-innen selbst wie Verschwörungstheorien klingen: Wenn wir zum Beispiel von angesehenen Philosophen lesen, die Pandemie sei nur erfunden worden, um einen Vorwand zu finden, den Ausnahmezustand auszurufen. Oft hören sich Verschwörungstheorien so an, als seien sie aus einem wohlbekannten Baukasten linker Kapitalismus- und Hegemoniekritik gestrickt: Etwas „Foucault“", eine Prise „Neoliberalismus“, dazu noch „Biopolitik“ - und schon haben wir eine feine Verschwörungssuppe angerichtet.

Kommt hier die pädagogische Besorgnis an ihre Grenzen? Oder wollen wir einem Philosophen vom Weltruf eines Giorgio Agamben schlichte Dummheit unterstellen, der erst die Existenz des Virus leugnete und, nachdem dies nicht mehr wirklich glaubhaft möglich war, verlautbaren ließ, das Problem sei, dass die Angst, das nackte Leben zu verlieren, die Menschen blind mache dafür, dass "die Schwelle, die die Menschlichkeit von der Barbarei trennt" (NZZ, 25.4.2020, S. 27) ${ }^{1}$ überschritten worden sei? Vielleicht begeht Agamben ja nur eine „,intelligente Dummheit" (Musil) - immerhin unterlegt er sein Raunen mit theoretischen Versatzstücken, die von ihm nur zu bekannt sind („Ausnahmezustand“, „Biopolitik“, „nacktes Leben“).

Offensichtlich erzeugt die Krise den Bedarf nach Kritik. Für Reinhart Koselleck war es noch umgekehrt gewesen. In seiner einflussreichen Schrift „Kritik und Krise“ (1959) hatte Koselleck geschrieben: „der kritische Prozess der Aufklärung hat die Krise [...] heraufbeschworen" (1959, S. 5). Das kritische Bewusstsein der Aufklärung und des sich bildenden Bürgertums hatte die politische Krise der Monarchie hervorgebracht: „Im Kreuzfeuer der Kritik wurde [...] die damals aktuelle Politik zer- 
mürbt“ (1959, S. 9). In der Zeit der Pandemie erzeugt hingegen eine durch das Virus ausgelöste „Krise“ den Bedarf nach „Kritik" an den herrschenden Umständen - sei es in Form der Verschwörungstheorie oder linker Gesellschaftskritik.

Ich bin Geograph, nicht Pädagoge. Meine Aufgabe ist nicht die „Belehrung“, sondern die „Kartierung“ - in diesem Fall die Kartierung diskursiver Landschaften. Die Motivation dieser Kartierungsübung liegt nicht in pädagogischer Besorgnis - ein Agamben lässt sich nicht belehren. Der Theoretiker weiß immer schon Bescheid - er kämpft um ,hermeneutische Vorsprünge“ (Vogl, MONOPOL, 9.4.2020). ${ }^{2}$ Stattdessen möchte ich - und folge hier dem Philosophen Hans Blumenberg - einen Raum für Nachdenklichkeit öffnen.

II.

Ausnahmezustand allenthalben. Bereits am 26.2.2020 meldete sich Giorgio Agamben in der italienischen Tageszeitung Il manifesto zu Wort ${ }^{3}$ : Medien und Behörden verbreiteten ein Klima der Panik (un clima di panico). Die Erfindung einer Epidemie (l'invenzione di un'epidemia) biete den ,idealen Vorwand“ (il pretesto ideale), einen Ausnahmezustand auszurufen und außergewöhnliche Maßnahmen über alle Grenzen hinaus auszudehnen. Am 19. März legte er in der NZZ nach: „Der Ausnahmezustand, auf den uns die Regierungen seit geraumer Zeit einschwören, ist längst zu unserem Normalzustand geworden“ (Agamben, NZZ, 19.3.2020, S. 29).

Agamben aktiviert hier einen Jargon des „Ernstfalls“, der seine Schriften schon seit langem durchzieht und der in den letzten Jahren in der akademischen Linken gerne aufgegriffen worden ist, so auch in der kritischen Geographie (vgl. Korf 2009). Zentral ist in diesem Jargon der Begriff des „Ausnahmezustands“. 1922 hatte der umstrittene Staatsrechtler Carl Schmitt apodiktisch geschrieben: „Souverän ist, wer über den Ausnahmezustand entscheidet" (Schmitt, [1922] 2004, S. 13). Für Schmitt gründet der Ausnahmezustand im Ereignis (der Entscheidung) und liegt deshalb „über“ dem Recht; für Agamben hingegen durchdringt der Ausnahmezustand das Recht: der Ausnahmezustand wird zur Regel (Agamben 2002, S. 48). Als Kronzeugen ruft Agamben hier Walter Benjamin in Erinnerung. In seinen „Geschichtsphilosophischen Thesen“ hatte Benjamin geschrieben: „Die Tradition der Unterdrückten lehrt uns darüber, dass der ,Ausnahmezustand', in dem wir leben, die Regel ist" (Benjamin 1965, S. 84; meine Hervorhebung).

Dieser Jargon durchzieht eine ganze Reihe weiterer Wortmeldungen meist männlicher Theoretiker (weshalb ich auch auf das Gender-Sternchen verzichte). Hans-Ulrich Gumbrecht sprach vom „Notstands-Staat“ (NZZ, 25.3.2020, S. 27). Peter Sloterdijk prophezeite eine "Securitoratie“ und eine „medico-kollektivistische Diktatur" (Le Point, 18.3.2020, $)^{4}$. Und Byung-Chul Han, Diagnostiker einer Müdigkeitsgesellschaft (Han 2010), schrieb: „Souverän ist, wer über Daten verfügt“. Han prognostizierte eine globale digitale Biopolitik, die „mit ihrem Kontroll- und Überwachungssystem sich unseres Körpers bemächtigt" (Die Welt, 17.4.2020).

Stichwort „Biopolitik“ - auch dies Teil des Jargons: Agamben, Gumbrecht, Sloterdijk und Han formulieren eine theoretisch begründete Unterscheidung zwischen dem „nackten“ und dem „guten“ Leben. Diese Unterscheidung macht Agamben in Homo Sacer (2002): Dort greift er die von Aristoteles vorgeschlagene Unterscheidung zwischen „nacktem“ Leben (zoë) und der politischen Existenz (bios) auf, um die Konstitution souveräner Macht in der Herstellung eines biopolitischen Körpers aufzuzeigen. Diese Unterscheidung kommt zum Ausdruck, wenn Agamben schreibt, die Gesellschaft sei angesichts der globalen Pandemie bereit, „praktisch alles zu opfern [...] die normalen Lebensbedingungen, die sozialen Beziehungen“. Auch bei Gumbrecht schwingt dies mit, wenn er warnt, „die Fülle des sinnlichen wie des sinnhaften Lebens [stehe auf dem Spiel]“ (NZZ, 18.4.2020, S. 27).

Byung-Chul Han rührt diesen Cocktail dann effektvoll zusammen. Aus der Müdigkeitsgesellschaft sei eine „Palliativgesellschaft“ geworden, die keinen Schmerz mehr ertrage und so das „gute“ Leben opfere: „Die Gesellschaft des Überlebens verliert ganz den Sinn für das gute Leben“ (Han 2020, S. 23, Hervorhebung im Original). Und Han setzt noch einen oben drauf: „Die Quarantäne ist eine virale Variante des Lagers, in dem das nackte Leben herrscht" (Han, 2020, S. 24). Mit dem Begriff „Lager“ ruft Han einen weiteren Kernbegriff des Jargons des Ernstfalls auf, hatte Agamben doch das Lager zum Signum der Moderne erklärt (Agamben 2002, S. 175 f.). Für Han resultiert daraus eine dystopische Vision: „Widerstandslos fügen wir uns dem Ausnahmezustand, der das Leben auf das nackte Leben reduziert" (Han, 2020, S. 24).

\section{III.}

Odo Marquard ist skeptisch: „Vernünftig ist, wer den Ausnahmezustand vermeidet" (Marquard, 2003, S. 260). Aber welcher Ausnahmezustand soll hier eigentlich vermieden werden? Es ist der diskursive Ausnahmezustand. Agamben, Gumbrecht, Han und Sloterdijk begeben sich in eine "Sonderzone Theorie“ (Sloterdijk, 2010, S. 126), von der aus eine „Hegemonie der Einsicht“ postuliert wird, aus der „die Einsicht in die Hegemonie" resultiert (Düttmann, 2004, S. 84). Theorie erhält so eine „intellektuelle Alarmfunktion“ (Encke, FAZ, 26.4.2020). ${ }^{6}$ Diese Haltung schwingt im diskursiven Ausnahmezustand mit, aber es scheinen die Sicherungen durchgebrannt zu sein. Kritik mutiert zur Hyper-Kritik. „Hyper-Kritik“, schreibt Thomas Edlinger, ist eine „zu Überreiztheiten und Selbstgerechtigkeit neigende Dynamik der Kritik" (Edinger, 2015, S. 48). Diese Form von Kritik überhöht die Theorie auf Kosten der Empirie. Die diskursiven Taktiken der Hyperkritik sind Dramatisierung und Moralisierung: „Man weiß schnell, wo man zu stehen hat" (Redepenning, 2007, S. 97). Das erklärt ihre Nähe zur Verschwörungstheorie.

Die heraufschwellende Bedrohungslage einer globalen Pandemie, die sich ab Mitte Februar 2020 immer mehr abzeichnete, erzeugte offensichtlich eine „Deutungsnot“ (Vogl, Monopol, 9.4.2020). In dieser Deutungsnot verspricht Theorie „hermeneutische Vorsprünge“(Vogl, ebd.). Ohne mühsame Umwege in die Empirie ermöglicht der kritische Jargon über seine Theoriesprache einen schnellen Durchgriff auf die Situation: man weiß immer schon, wie die Welt eigentlich funktioniert. Doch zum Zeitpunkt, als sich Agamben, Gumbrecht, Han und Sloterdijk zu Wort meldeten, gab es noch wenig gesicherte Informationen über den Virus und seine Ausbreitungsformen. So entstehen Erklärungen für einen Sachverhalt, „der keineswegs festgestellt war" (Vogl, FAZ, 26.4.2020) ${ }^{7}$. 
Kritische Theorie wird zu einer defizitären Form von Zeitdiagnostik, wenn sie sich dem Sog der Beschleunigung hingibt, die die Deutungsnot erzeugt. Zeitdiagnostik, schreibt Julia Lossau, trifft „leichtfertige Antworten auf die Frage, in welcher Zeit oder in welcher Gesellschaft wir ,eigentlich ' leben “ (Lossau, 2001, S. 238). Kritische Theorie verkommt dann zu einem "Jargon der Eigentlichkeit" - einen Begriff, den Theodor W. Adorno eigentlich für andere - reaktionäre - Formen des Denkens über die „Eigentlichkeit“ des „Daseins“ reserviert hatte: Martin Heidegger. Adorno schreibt: „Dass die Jargonworte, unabhängig vom Kontext wie vom begrifflichen Inhalt, klingen, wie wenn sie ein Höheres sagten, als was sie bedeuten, wäre mit dem Terminus Aura zu bezeichnen“ (Adorno, 1964, S. 11 f.). Was Adorno zu Heidegger sagt, trifft sicher auch die Aura von Agambens oder Hans Schriften. Nicht zufällig sind beide auch treue Anhänger des Meisters.

Es ist die Aura des raunenden und warnenden Meisterdenkers, der gerne von in die Jahre gekommenen Philosophen gepflegt wird. Schon seit längerem gibt es einen theoretischen Erregungszustand, der vor den Gefahren der Gefahrenabwehr warnt und die Dystopie eines Überwachungsstaates kolportiert. Die theoretischen Referenzen blieben dabei über Jahre die gleichen: Carl Schmitts „Ausnahmezustand“ wird mit Walter Benjamin emanzipatorisch gewendet und mit einer Prise Foucault („Biopolitik“, „Panoptikum“) gewürzt. Aufgrund des diagnostizierten politischen Ausnahmezustands wird ein intellektueller „Außerordentlichkeitsbedarf“ (Marquard 2000) ausgerufen. Die Kritikerin fungiert dann „als Ankläger[in], als oberste Gerichtsinstanz [...] und als Partei zugleich" (Koselleck, 1959, S. 6). Kritik, so hatte es Michel Foucault formuliert, sei „die Kunst nicht auf diese Weise und um diesen Preis regiert zu werden" (Foucault, 1992, S. 12). Die Meisterdenker nehmen für sich in Anspruch, sich gegen die „Uneigentlichkeit“ heutiger Regierungsformen zu wehren.

Die Theorie der Meisterdenker wird zu einer Art „anschwellendem Bocksgesang“ (Strauss, 1993), der im „Mysterienlärm des Unheils“ erstarrt. In seinem umstrittenen Essay, 1993 im SPIEGEL veröffentlicht, hatte der Dramaturg Botho Strauss, selbst nicht vor der Aura des raunenden Meisterdenkers gefeit, der Hoffnung Ausdruck gegeben, „dass ein Satz, den angeblich Max Frisch zu einem Kollegen gesagt hatte ,Werde im Alter nicht weise, sondern bleibe zornig' -, als der Gemeinplatz kritischer Bequemlichkeit erkannt wird, der er in Wahrheit ist“ (1993, S. 206). Die Bequemlichkeit des Zorns erlaube Auslassungen: „Was darf er alles außer Acht lassen, um seinen Zorn zu konservieren“ (ebd.).

\section{IV.}

Was hilft gegen die Deutungsnot der Krise und dem anschwellenden Bocksgesang der Meisterdenker? Das Wesen des kritischen Denkens liegt nicht im Urteil, so Marcus Quent (2019, S. 39), „sondern in der, Unterbrechung ' des Urteils“. Unterbrechung ermöglicht Nachdenklichkeit: Nachdenklichkeit, so der Philosoph Hans Blumenberg (1981, S. 57), bedeute abzuwarten, „was sich jeweils noch zeigt“. Sie verschont die Theoretikerin vor der „Geste der Überbietung“ (Quent, 2019, S. 41), dem „Rausch des Epochalen“ (Vogl), dem „Außerordentlichkeitsbedarf" (Marquard) und dem „Jargon der Eigentlichkeit“
(Adorno). Nachdenklichkeit ermöglicht eine Kritik, „die ihre eigene Generalisierungstendenz unterbricht“ (Vogl, 2008, S. 115). „Nachdenklich ist, wer den Ausnahmezustand vermeidet“, schreibt Benjamin Dober (2019, S. 294).

Nachdenklichkeit erfordert „Erschütterungsbereitschaft" (Amlinger und Gess, 2020): Erschütterungen in der Lebenswelt - Krisen - sollten mit einer Erschütterung der Theorie einhergehen - durch „Wahrnehmungsvorbehalte" (Vogl) und eine „Provinzialisierung des Blickes“. Als „Provinzialisierung “ hat sich in der Postcolonial Theory dank der wegweisenden Arbeiten von Dipesh Chakrabarty eine Haltung eingebürgert, im Blick auf den vermeintlichen Universalismus westlicher Kategorien („Menschenrechte“) und Geschichtenerzählungen („Fortschritt") deren kontingente Entstehungsbedingungen aufzuzeigen - und deren Kosten (Chakrabarty 2010). „Provinzialisierung " bedeutet eine Akzentverschiebung: die eigene Kontingenz in den Blick zu bekommen und zugleich über den eigenen Tellerrand hinauszuschauen. Gerade der intellektuelle Ausnahmezustand der Theorie verdeckt jedoch die Idiosynkrasien der eigenen Diagnosen und verhindert damit Akzentverschiebungen.

Als Anhaltspunkt, was damit gemeint ist, empfehle ich eine Lektüre des berührenden und zugleich schockierenden Berichts von Arundhati Roy „Durch das Tor des Schreckens“ (DIE ZEIT, 8.4.2020, S. 4 f.) und kontrastiere diesen mit ByungChul Hans jüngstem Buch zur Palliativgesellschaft (Han 2020). Roy schildert in ihrer Reportage die furchtbare Verzweiflung Millionen verarmter, hungriger, durstiger Menschen in Indien, die nach der Verkündigung eines „Lockdowns“ innerhalb kürzester Zeit gezwungen wurden, aus den Städten zurück aufs Land zu gehen. Diese marginalisierten Tagelöhner wurden wie unerwünschte Substanzen vertrieben. Zuhause auf dem Land wartete nicht nur das Virus, sondern der Kampf ums nackte Überleben: „ihnen war klar, dass sie zu Hause womöglich langsam verhungern würden“, schreibt Roy dazu.

Han schreibt zur Palliativgesellschaft, in der Quarantäne erstarre „das Leben ganz zum Überleben“ (Han, 2020, 23). Han (2020, S. 26) legt dann noch einmal nach: „Die von der Hysterie des Überlebens beherrschte Gesellschaft ist eine Gesellschaft der Untoten“. Das soll tief klingen - nach (Un-)Eigentlichkeit vermutlich. Aber vergreift sich Han nicht im Genre? Übersieht er nicht einen ganz anderen, existentiellen Ausnahmezustand, der in den marginalisierten Randzonen postkolonialer Gesellschaften wie Indien zum Alltag gehört, durch den „Lockdown“ aber noch einmal in seiner existentiellen Dimension dramatisiert wurde? Hier geht es um das „nackte Überleben“ nicht nur in einem metaphorischen Sinne, wie das in der Theoriesprache zum Ausdruck kommt. Dabei kommt eine ganz andere, existentielle Dimension „nackten Überlebens" zum Ausdruck als im erzwungenen Homeoffice in Deutschland, auch wenn für Han (2020, S. 24) die Quarantäne zur „virale[n] Variante des Lagers [wird], in dem das nackte Leben herrscht" (Theorie ist auch eine Frage des guten Geschmacks.).

Epistemologisch interessant ist auch, dass Han nicht expliziert, wo es diese Palliativgesellschaft eigentlich geben soll: in Deutschland, in Europa, weltweit? Es ist genau diese mangelnde Situierung der eigenen Diagnose, die eine „Provinzialisierung " benötigt. Von der eigenen Lebenssituation wird generalisiert, aber nicht theoretisch abstrahiert im Sinne einer 
Idiosynkratisierung: Han schreibt seine Zeitdiagnose in einem universalistischen Gestus, ohne die Kontingenz der eigenen Erfahrung und Generalisierung zu reflektieren. Es wirkt schon fast absurd, wenn er „asiatischen“ Staaten „Systemvorteile bei der Eindämmung der Pandemie“ (Han, Die Welt, 17. April 2020) unterstellt, da sie in der digitalen Biopolitik schon weiter seien als „Europa“. Die Abstraktionsebene ist hier sehr grobmaschig. Aus dieser Sicht wird Roys Reportage zu Indiens Tagelöhnern zu einem Spezialfall, der keiner besonderen Aufmerksamkeit bedarf.

Der Jargon der Eigentlichkeit, den Agamben und Han mit Hilfe des Vokabulars von „Ausnahmezustand“, „nacktes Leben“, „Lager“, und „Biopolitik“ hervorbringen, agiert in einer Denkstimmung des „Ernstfalls“: Die liberale Demokratie scheint grundlegend - in ihrer Existenz - in Gefahr zu sein, ebenso das "gute“ Leben. Implizit wird hier ein Bild eines großen Plans einer biopolitischen Machtübernahme gezeichnet, die eigentlich schon lange im Untergrund im Gang und durch die Pandemie nun in ihrer Intensität sichtbarer geworden sei. Die Empirie wird gar nicht mehr benötigt, da die „wirklichen“ $\mathrm{Zu}$ sammenhänge hinter dem Schein der Wirklichkeit den Meisterdenkern der Theorie ja schon vorher klar ist. Erschüttert ist der Meisterdenker nicht, allenfalls bestätigt.

„Erschütterungsbereitschaft" erfordert andere Formen der Theorie - eine Theorie, die sich von der Vielfalt der Lebenswelt noch irritieren lässt und Krisenphänomene nicht immer schon als Bestätigung der eigenen Theorie subsumiert. Eine Frage, die sich in der konkreten Situation der Pandemie stellt, wäre zum Beispiel, welche Begriffe hilfreich sind, die politischen Maßnahmen zur Eindämmung der Pandemie als Herrschaftsform und rechtliche Praxis zu beschreiben. Vielleicht haben wir es in der Pandemie gar nicht mit einem „Ausnahmezustand“ zu tun, wenn damit eine existenzielle Entscheidung über die souveräne Macht (Carl Schmitt) gemeint sein soll.

So argumentiert z. B. der Kulturwissenschaftler Jan Söffner (NZZ, 14.6.2020) ${ }^{8}$, Schmitts Theorie des Ausnahmezustands sei „nur teilweise tauglich“, den rechtlichen Raum, in dem über die Pandemiemaßnahmen entschieden wurde, zu kartieren. Aus juristischer Sicht wurden mit den Pandemiemaßnahmen keine Rechtsordnung außer Kraft gesetzt, sondern lediglich einige Grundrechte durch Rechtsverordnungen vorüber eingeschränkt. „Souveränität“ (die Kategorie, die bei Schmitt im Zentrum steht), so Söffner, sei hier eine irreführende Kategorie. Weder in Deutschland, noch in der Schweiz, habe es sich bei den Corona-Maßnahmen um eine „Selbstermächtigung der Exekutiven“ gehandelt. Vielmehr wurden die Exekutiven in diesen „Notrechtszustand“ hineingedrängt und die Übernahme exekutiver Verantwortung wurde eher zur Last als zur Lust: So sagte die Schweizer Bundespräsidentin, Simonetta Sommaruga, in einem Interview (NZZ vom 19.4.2020)9 „Der Bundesrat ist froh, wenn das Parlament tagt“. Er, der Bundesrat, wolle „nicht so weiterregieren“. Schon am 29.4.2020 beschließt der Bundesrat, die zur Bekämpfung der Pandemie erlassenen Notverordnungen in ein befristetes Bundesgesetz umzuwandeln, das er am 19.6.2020 in die Vernehmlassung schickt. Auch die latente Überforderung der Schweizer Kantone, ein effektives "contact tracing“ $\mathrm{zu}$ installieren, lassen Byung-Chul Hans panoptische Phantasie einer allumfassenden Überwachung eher lächerlich klingen.
Es wird offensichtlich, dass wir ein sehr viel differenzierteres Begriffsvokabular benötigen als „Ausnahmezustand“, „Biopolitik“, „nacktes Leben“, „Souveränität“ und „Ermächtigung“. Philipp Sarasin (2020) stellte ganz offen die Frage, ob man mit Foucault die Pandemie besser verstehen könne und beantwortet die selbstgestellte Frage skeptisch: Der Begriff der Biopolitik helfe nicht weiter, um die Pandemie zu verstehen, auch wenn die Situation als „biopolitischer Traum“ erscheine. Und Sarasin wendet sich explizit gegen „kritische Kritiker“, wie Giorgio Agamben, „die mit ,Foucault' im Gepäck nun zu durchschauen meinen, was gerade geschieht". Stattdessen sollten wir uns auf die tastende Suche nach alternativen Begriffen aufmachen. Für Henning Trüper, zum Beispiel zeigt sich eine „Unsouveränität“ in der Pandemie (Trüper, 2020). Mit „Unsouveränität“ bezeichnet Trüper eine Antipolitik als „Ostentation von Handlungsmacht in einer Lage grundsätzlicher Ohnmacht" (ibid.). Souverän sei hier allenfalls die humanitäre Moral („Leben retten“). Für Trüper sind denn auch Agamben \& Co. die „uneigentlichen Ärzte [der Gesellschaftskritik] an der Seite der eigentlichen, [die in den Intensivstationen arbeiten]".

\section{V.}

Letztlich folgen Agamben und Han in ihrer Kritik an den Pandemiemaßnahmen einer „Hermeneutik des Verdachts“ (Ricoeur, 1974, S. 45 ff.), die für sich in Anspruch nimmt, einen Schein der Dinge zu entlarven, der den Subjekten (außer den Kritiker/-innen) verborgen bleibt. Die Kritikerin spricht den Verdacht aus: Agamben und Han verdächtigen die liberale Demokratie: ihre Liberalität sei nur vorgetäuscht - eben ein „Schein“. Deshalb sei es Aufgabe der Kritikerin, Alarm zu schlagen, den Schein aufzudecken, die „schlafenden Subjekte“ aufzuwecken - ihnen die „Uneigentlichkeit“ der demokratischen Institutionen vor Augen zu führen. So werden Alarmismus und Angstmache zu einer Methode der „linken“ Kritik die Grenzen zur Verschwörungstheorie weichen zunehmend auf.

Treibt es diese „Hermeneutik des Verdachts“ zu bunt und wild, wird es Zeit, den „Dämonenräumdienst“ (Marcel Beyer) zu rufen. Dieser Dämonenräumdienst ist gegen die Schimäre des Verdachts gerichtet und versteht sich deshalb als „eine Kritik rettende Kritik“ (Figal, 2009, S. 352). Der „Dämonenraumdienst" arbeitet sich am problematischen Gestus des Verdachts ab. Er unterbricht den „Mysterienlärm des Unheils“ ebenso wie den theoretischen „Universalisierungsehrgeiz" (Bauer, 2011, S. 251). Er mistet das Begriffsvokabular aus. Der Dämonenräumdienst vollzieht damit die „pädagogische“ (oder „therapeutische“) Aufgabe einer Kritik der Kritik in der Krise. 


\section{Anmerkungen}

1 https://www.nzz.ch/feuilleton/coronavirus-giorgio-agamben-zum-zusammenbru ch-der-demokratie-ld.1551896 (online am 15.4.2020 erschienen; in der gedruckten Ausgabe erst am 25.4.2020).

2 Interview mit dem Magazin MONOPOL, 9.4.2020: https://www.monopol-mag azin.de/joseph-vogl-coronakrise

3 https://ilmanifesto.it/lo-stato-deccezione-provocato-da-unemergenza-immotiva tal

4 https://www.lepoint.fr/politique/sloterdijk-le-systeme-occidental-va-se-reveler-au ssi-autoritaire-que-celui-de-la-chine-18-03-2020-2367624_20.php

5 https://www.welt.de/kultur/plus206681771/Byung-Chul-Han-zu-Corona-Vern unft-nicht-dem-Virus-ueberlassen.html

$6 \mathrm{https} / /$ www.faz.net/aktuell/feuilleton/debatten/corona-theorien-warum-wir-lieb er-drosten-als-sloterdijk-hoeren-16741391.html

7 Vogl wird zitiert in einem Beitrag von Cornelia Encke: https://www.faz.net/aktuell /feuilleton/debatten/corona-theorien-warum-wir-lieber-drosten-als-sloterdijk-ho eren-16741391.html

8 https://www.nzz.ch/feuilleton/coronakrise-sind-wir-unterwegs-zu-platons-waech terstaat-ld.1560404

9 https://www.nzz.ch/schweiz/bundespraesidentin-sommaruga-das-virus-haelt-sich -nicht-an-plaene-ld.1552278

\section{Literatur}

Adorno, T. W. (1964). Jargon der Eigentlichkeit. Zur deutschen Ideologie. Frankfurt a. M.: Suhrkamp.

Agamben, G. (2002). Homo Sacer. Die souveräne Macht und das nackte Leben. Frankfurt a. M.: Suhrkamp.

Amlinger, C. \& Gess, N. (2020). reality check: Wie die Corona-Krise kritische und weniger kritische Theorien auf den Prüfstand stellt. Geschichte der Gegenwart. Zugriff am 17.09.2020 https://geschichtedergegenwart.ch/reality-check-wie-die-corona-kr ise-kritische-und-weniger-kritische-theorien-auf-den-pruefstand-stellt/

Bauer, Thomas (2011). Kultur der Ambiguität. Berlin: Verlag der Weltreligionen.

Benjamin, W. (1965). Zur Kritik der Gewalt und andere Aufsätze. Mit einem Nachwort von Herbert Marcuse. Frankfurt a. M.: Suhrkamp.

Beyer, M. (2020). Dämonenraumdienst: Gedichte. Frankfurt a. M.: Suhrkamp.

Blumenberg, H. (1981). Nachdenklichkeit: Dankrede. Jahrbuch der Deutschen Akademie für Sprache und Dichtung (II), 51-61.

Chakrabarty, D. (2010). Europa als Provinz. Perspektiven postkolonialer Geschichtsschreibung. Frankfurt a. M.: Campus.

Dober, B. (2019). Ethik des Trostes. Hans Blumenbergs Kritik des Unbegreiflichen. Weilerswist: Velbrück. https://doi.org/10.5771/9783748906612
Düttmann, A. G. (2004). Philosophie der Übertreibung. Frankfurt a. M.: Suhrkamp. Edlinger, T. (2015). Der wunde Punkt. Vom Unbehagen an der Kritik. Berlin: Suhrkamp.

Figal, G. (2009). Verstehen - Verdacht - Kritik. In R. Jaeggi \& T. Wesche (Hrsg.). Was ist Kritik? (S. 339-352). Frankfurt a. M.: Suhrkamp.

Foucault, M. (1992). Was ist Kritik? Berlin: Merve.

Han, B.-C. (2020). Palliativgesellschaft: Schmerz heute. Berlin: Matthes \& Seitz.

Han, B.-C. (2010). Müdigkeitsgesellschaft. Berlin: Matthes \& Seitz.

Korf, B. (2009). Geographie des Ernstfalls. Geographische Zeitschrift 97 (2,3), 151167.

Koselleck, R. (1959). Kritik und Krise. Eine Studie zur Pathogenese der bürgerlichen Welt. Frankfurt a. M.: Suhrkamp.

Marquard, O. (2003). Zukunft braucht Herkunft. Philosophische Essays. Stuttgart: Reclam.

Marquard, Odo (2000). Philosophie des Stattdessen. Philosophische Essays. Stuttgart: Reclam.

Quent, M. (2019). Das Delirium der Kritik. Zeitschrift für Ideengeschichte XIII (1), 33-42. https://doi.org/10.17104/1863-8937-2019-1-33

Redepenning, M. (2007). Die Moral der critical geopolitics. Geographische Zeitschrift 95 (1,2), 91-104.

Ricoeur, P. (1974). Die Interpretation. Ein Versuch über Freud. Frankfurt a. M.: Suhrkamp.

Sarasin, P. (2020). Mit Foucault die Pandemie verstehen? Geschichte der Gegenwart Zugriff am 17.09.2020 https://geschichtedergegenwart.ch/mit-foucault-die-pandemie-verstehen/

Schmitt, C. (1922/2004). Politische Theologie: Vier Kapitel zur Lehre von der Souveränität. Berlin: Duncker \& Humblot.

Sloterdijk, P. (2010). Scheintod im Denken. Von Philosophie und Wissenschaft als Übung. Berlin: Suhrkamp.

Strauss, B. (1993). Anschwellender Bocksgesang. Der Spiegel 93(6), 202-207.

Trüper, H. (2020). Unsouveränität in der Pandemie. Zugriff am 17.09.2020 https:// www.zflprojekte.de/zfl-blog/2020/03/24/henning-trueper-unsouveraenitaet-in-der -pandemie

Vogl, J. (2008). Über das Zaudern. Zürich: Diaphanes.

\section{Benedikt Korf}

ist Professor für Politische Geographie an der Universität Zürich. Sein Forschungsschwerpunkt ist die geographische Konfliktforschung mit regionalen Schwerpunkten in Südasien und dem Horn von Afrika. 